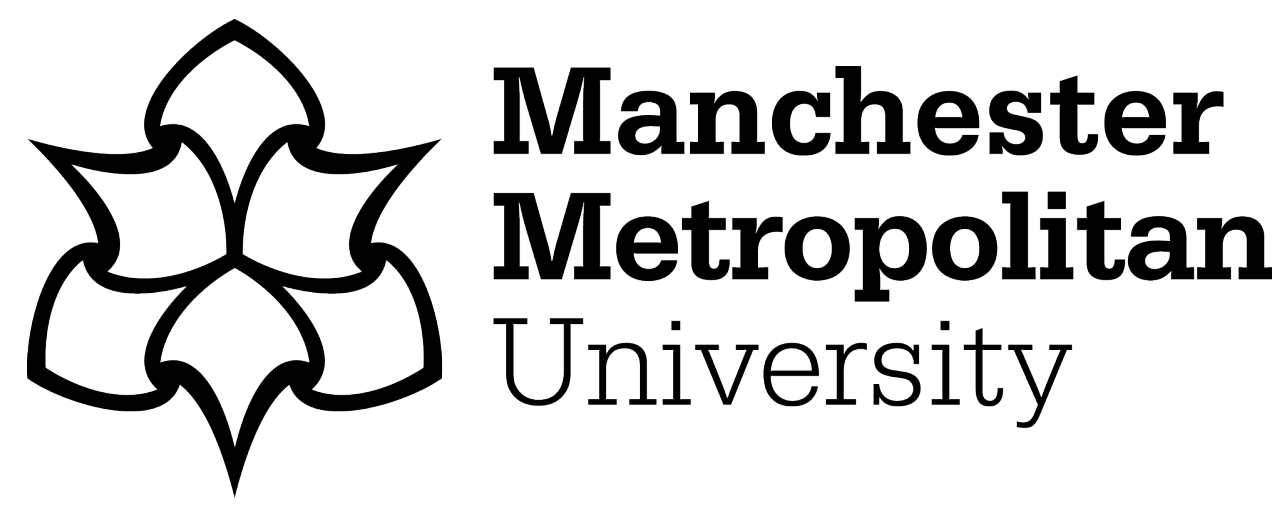

Owda, AYE, Salmon, Neil, Rezgui, Nacer and Shylo, Sergiy (2017) Millimetre wave radiometers for medical diagnostics of human skin. In: 2017 IEEE SENSORS, 30 October 2017 - 01 November 2017, Glasgow, United Kingdom.

Downloaded from: https://e-space.mmu.ac.uk/620712/

Version: Accepted Version

Publisher: IEEE

DOI: https://doi.org/10.1109/ICSENS.2017.8234132

Please cite the published version 


\title{
Millimetre Wave Radiometers for Medical Diagnostics of Human Skin
}

\author{
Amani Yousef Owda ${ }^{1}$, Neil Salmon ${ }^{1}$, Nacer Ddine Rezgui ${ }^{1}$, and Sergiy Shylo ${ }^{2}$ \\ ${ }^{1}$ Dep. Electrical and Electronic Engineering, Manchester Metropolitan University, Manchester, United Kingdom \\ ${ }^{2}$ Usikov Institute of Radiophysics and Electronics National Academy of Sciences of Ukraine, Kharkiv, Ukraine
}

Email:amaniabubaha@gmail.com,n.salmon@mmu.ac.uk,n.rezgui@mmu.ac.uk,shilo@ire.kharkov.ua

\begin{abstract}
A technique to measure the human skin emissivity in vivo is described for the frequency band 80-100 GHz. Emissivity measurements were performed on 60 participants, 35 males and 25 females, with ages ranging from 20 to 60 years. Results show that the emissivity of males is higher than that of females. The study suggests a trend in the emissivities with age and gender, which might be due to variations of skin thickness and water content. As non-contact screening is desirable in medical applications, passive millimeter-wave sensing could be a means of achieving this in the diagnosis of skin disease or damage, where the disease/damage alters the water content or the skin thickness.
\end{abstract}

Keywords—radiometric; emissivity; millimeter wave.

\section{INTRODUCTION}

Over the past few decades, millimetre wave technology has shown a steady and consistent development, with a wide variety of devices and system architectures (imaging, non-imaging, radiometers and radars) becoming available. As an imaging sensor, these systems can deliver spatial resolutions down to around half of the wavelength of the radiation used, $\sim 1.0 \mathrm{~mm}$ for the millimetre wave band. This property enables highly localized, non-contact measurements to be made just below the skin surface, a capability not available in the visible spectrum. A convenient metric to describe the condition of human skin is the emissivity [1], this being defined as the ratio of the level of thermal electromagnetic (Planck) radiation to that level from a thermal blackbody source at the same thermodynamic temperature [2]. Many studies reveal strong correlation between skin dielectric properties, skin thickness, water content and hydration level, all of which vary with age, gender and body site [3-5]. However, to date the potential for medical diagnostics using this information has not been exploited, possibly because insufficient attention has been paid to experimental detail. This study redresses this balance by analyzing the emissivity of skin at different regions of the human body, for subjects of different gender, ethnicity and age, over the frequency band (80-100) GHz.

\section{EXPERIMENTAL SETUP}

\section{A. Radiometric Calibration and Emissivity Measurements}

A radiometer operating over the frequency band 80-100 $\mathrm{GHz}$ was used to measure the human skin emissivity. The equipment for measurement and calibration comprises: a horn antenna connected to Millimeter-Wave Monolithic Integrated Circuit (MMIC) detector, two pieces of carbon loaded foam absorbers acting as hot and cold calibration sources, a digital voltmeter and the subject tissue to be measured, as illustrated in Fig. 1. The horn antenna has a rectangular aperture $\left(3 \times 2.5 \mathrm{~cm}^{2}\right)$ and a nominal gain of $20 \mathrm{dBi}$ over the frequency band (80-100) GHz. The MMIC detector consists of a two-stage low-noise amplifier (LNA), zero bias detector and buffer amplifier. The radiometer is calibrated by measuring emissions from the hot and cold sources $[1,6]$ at respective temperatures of $\mathrm{T}_{\mathrm{H}}$ and $\mathrm{T}_{\mathrm{C}}$.

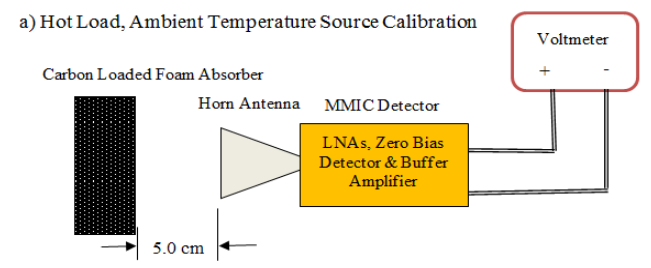

b) Cold Load, Liquid Nitrogen Source Calibration $\left(\mathrm{LN}_{2}\right)$

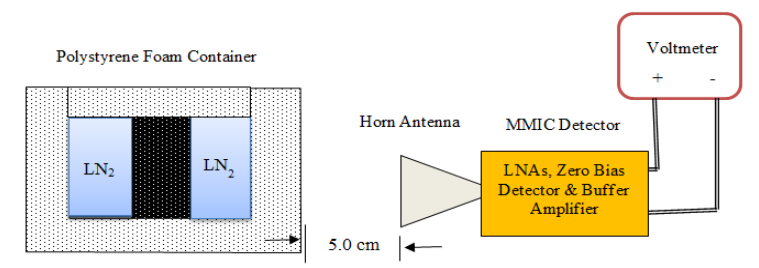

c) Human Skin Measurements

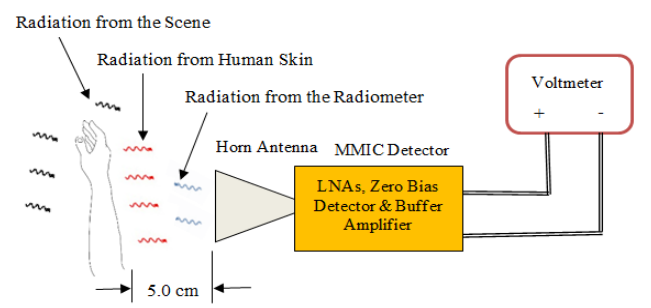

Fig. 1. Experimental setup for human skin emissivity measurements.

Manchester Metropolitan University's Internal Investment and Innovation Fund and Engineering and Physical Sciences Research Council (EPSRC) have funded this work. 
The horn antenna may be located at a distance $(\sim 5.0 \mathrm{~cm})$ from three different radiation sources: 1) "Hot" black body, a piece of carbon loaded foam absorber with $\mathrm{T}_{\mathrm{H}}=\mathrm{T}_{\text {ambient }}=293 \mathrm{~K}$, 2) "Cold" black body, a piece of carbon loaded foam absorber is dipped in the liquid nitrogen with $\mathrm{T}_{\mathrm{C}}=77 \mathrm{~K}$ and 3 ) the human skin. The system response is assumed to be linear, since the measurements are done indoors and in an anechoic environment. The output of the receiver for "Hot" black body, Fig. 1a, can be expressed as $[1,6]$ :

$$
V_{H}=\alpha\left(T_{H}+T_{N}\right)
$$

Where, $T_{H}$ is the hot load temperature in $\mathrm{K}, \alpha$ is the receiver responsivity in $\mathrm{V} / \mathrm{K}$, and $\mathrm{T}_{\mathrm{N}}$ is the receiver noise temperature in K. For the "Cold" black body, the output of the receiver, Fig. 1b, is $[1,6]$ :

$$
V_{C}=\alpha\left(T_{C}+T_{N}\right)
$$

The radiation temperature of the skin can be expressed in terms of the skin emissivity $\eta$, the skin thermodynamic temperature $\mathrm{T}_{\mathrm{S}}$, and the background illumination temperature $\mathrm{T}_{0}[6]$ :

$$
T_{b}=(1-\eta) T_{0}+T_{s} \eta
$$

From (1) to (3), and identifying $\mathrm{T}_{0}$ with $\mathrm{T}_{\mathrm{H}}$, the measured emissivity of the human skin can be expressed as:

$$
\eta=\frac{\left(V_{S}-V_{H}\right)\left(T_{H}-T_{C}\right)}{\left(T_{S}-T_{H}\right)\left(V_{H}-V_{C}\right)}
$$

A digital voltmeter with $0.1 \mathrm{mV}$ resolutions was used to measure the output voltage for the target area of the skin, Vs, and an infrared thermometer with absolute accuracy $0.01{ }^{\circ} \mathrm{C}$ was used to measure the skin surface temperature, Ts.

\section{RESULTS AND DISCUSSION}

The objective of these experiments is to study how the emissivity of the human skin varies with age and gender. The measurements were performed on 60 healthy participants; 35 males and 25 females. Participants were divided into four age groups: $20-30,30-40,40-50$, and 50-60. There were 5-10 subjects in each group.

\section{A. Radiometric Calibration Results}

The calibration Y-factor, defined as the ratio of receiver output when measuring the hot black body source, to that measuring the cold source was 1.408 . This gives a receiver noise temperature of $453.7 \mathrm{~K}$.

\section{B. Male Emissivity Measurements}

The measurements in Figs. 2, and 3 represent the mean emissivities for the male groups, with error bars representing the standard error in the mean (SEM). The measurements show significant and consistent variations in emissivity at different locations of the hand for different age groups. Emissivity for dorsal surface and outer wrist positions are higher than volar side and inner wrist positions. Skin on volar side and inner wrist is thin and smooth [7], and as a result, the blood vessels are closer to the skin surface. This increases the reflectivity of the skin and makes the emissivity lower as a result of conservation of energy $[6,8]$. However, the skin on the dorsal surface of the forearm and outer wrist is thick and dense [7] and this makes the skin reflectivity lower and as a result skin emissivity increases. The results also suggest two age-related trends: 1) a possible slight decrease in the emissivity in the age groups between 20-40 years. This decrease is consistent with the fact that male skin thickness decreases gradually with the age [3, 8]. 2) An increase in the emissivity in the age groups between 40-60 years. This increase is consistent with the fact that water content and skin hydration level decrease with the age $[4,5]$ and this makes aged skin drier compared with young skin [3]. The statistical analysis of the male samples shows that the sample mean $(\mu)$ of the differences in the emissivities between the dorsal and volar side of the forearm is 0.0675 with a sample standard deviation $(\sigma)$ of 0.0319 , whereas the sample mean of the differences in the emissivities between the inner and outer wrist is 0.0535 with a sample standard deviation of 0.0336 . These results indicating a very consistent difference between the emissivity of different location on the body. Error propagation through (4) indicates the uncertainty on the measured emissivity is \pm 0.002 .

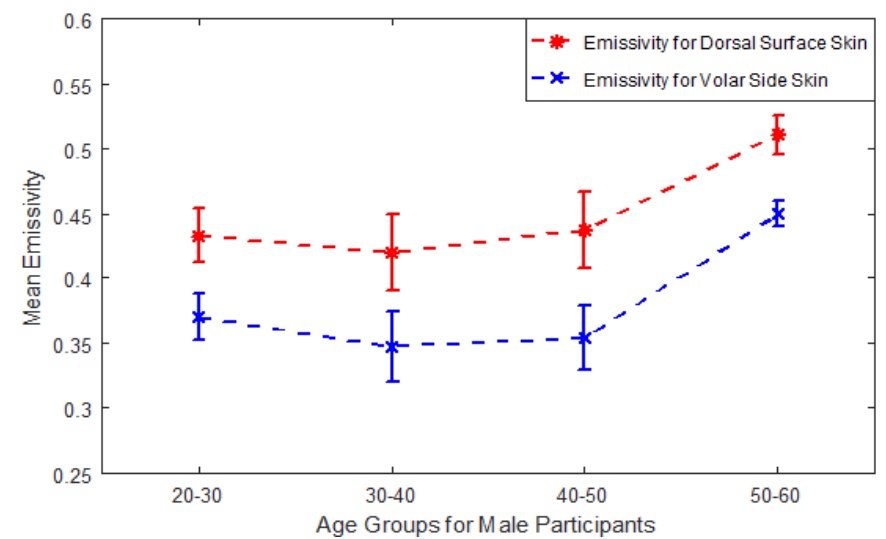

Fig. 2. Dorsal surface and volar side emissivity measurements for 35 male, with standard error in the mean $(\mathrm{SEM} \leq 0.029)$ for each age group.

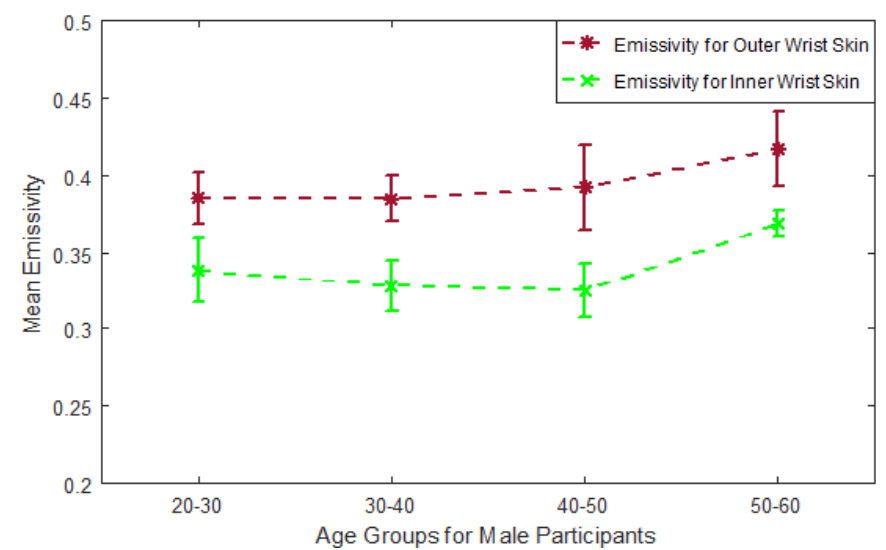

Fig. 3. Outer and inner wrist emissivity for 35 male, with standard error in the mean $(\mathrm{SEM} \leq 0.027)$ for each age group. 


\section{Females Emissivity Measurements}

The measurements in Figs. 4 and 5 show that emissivity of the dorsal surface skin is higher than that of the volar side and emissivity of the outer wrist is higher than that of the inner wrist for all female groups. The measurements also suggest two agerelated trends: 1) relatively unchanged emissivity in the age groups 20-50 years with slight increase in dorsal surface skin emissivity. 2) Significant decreases in the emissivity in the age group 50-60 years. These trends are consistent with the fact that females skin maintain the same thickness up to the age of 50 and then beyond this the skin thickness gets significantly thinner, probably due to decreased oestrogen levels after menopause [3]. The statistical analysis of the female sample shows that, the sample mean of the differences in the emissivities between the dorsal surface and the volar side locations is 0.0678 with a sample standard deviation of 0.0439 , whereas the sample mean of the differences in the emissivities between the inner and outer wrist location is 0.0645 with a standard deviation of 0.0387 .

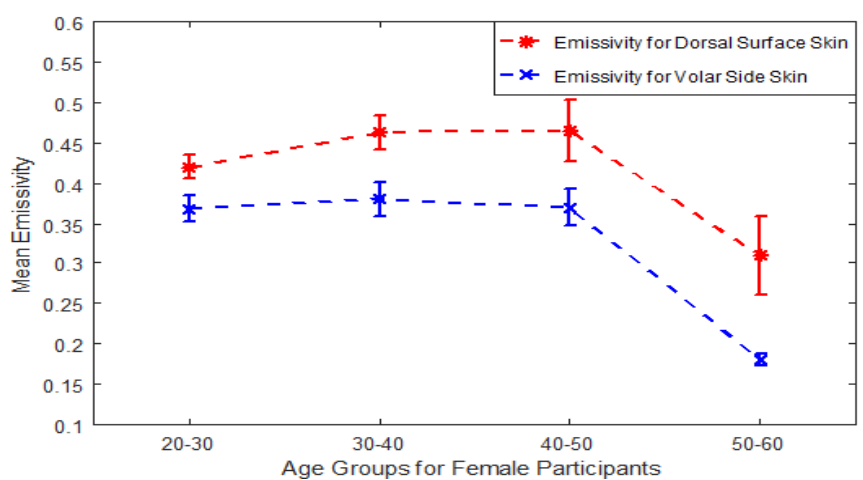

Fig. 4. Dorsal surface and volar side emissivity for 25 female, with standard error in the mean $(\mathrm{SEM} \leq 0.038)$ for each age group.

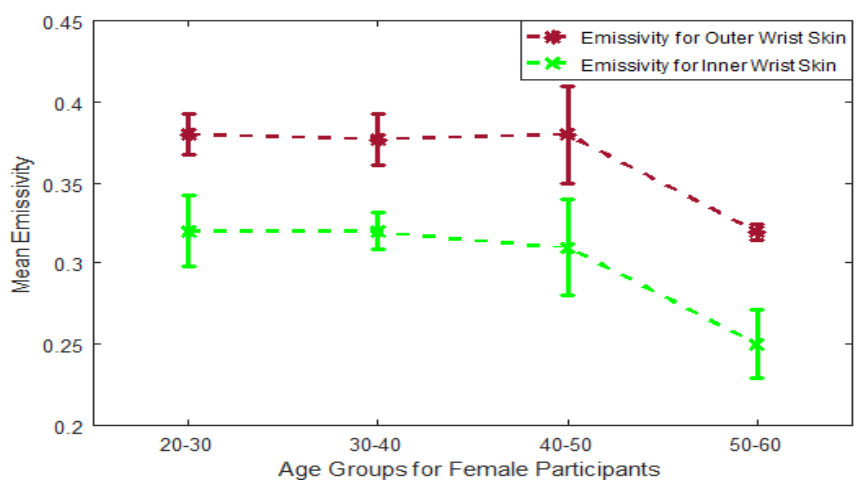

Fig. 5. Outer and inner wrist emissivity for 25 female, with standard error in the mean $(\mathrm{SEM} \leq 0.04)$ for each age group.

\section{Comparison Between Males and Females Emissivity}

The measurements in Fig. 6 show that, when averaged over all age groups, the emissivity of males is higher than that of females in all positions with a difference in the standard error in the mean of approximately 0.02 . This result is consistent with the fact that the skin of men is thicker than that of women $[3,8]$. Furthermore, the measurements show that for both genders, the emissivity is higher in the locations where the skin is thick, and it is lower in the locations where the skin is thin. The emissivity measurements in this paper are consistent with [6].

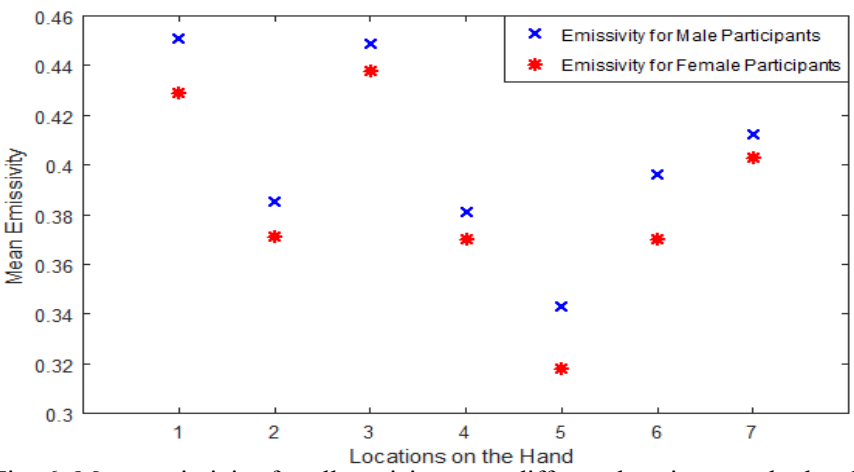

Fig. 6. Mean emissivity for all participants at different locations on the hand: 1) palm of hand 2) back of hand 3) dorsal surface of the forearm 4) volar side of the forearm 5) inner wrist 6) outer wrist and 7) elbow.

\section{E. Exploitation in Medical Applications}

As human skin emissivity shows strong correlation with skin thickness and water contents, this indicates that radiometry could be used as a non-contact technique to detect and monitor skin conditions such as psoriasis, eczema, malignancy and burn wounds.

Although measurements presented here are made using a single channel radiometer, an exploitation route might consider the development arrays of antennas to make measurements on larger skin areas or perhaps the whole of the human body in tens of seconds.

\section{CONCLUSION}

The measurements show variations in emissivity between skin location, gender and age group. The results indicate that the emissivity of human skin in the millimeter wave band is rich in information and this could potentially be exploited for the diagnosis of a range of medical conditions.

\section{REFERENCES}

[1] F. T. Ulaby, R. K. Moore, A. K. Fung, Microwave remote sensing: active and passive, volume I: microwave remote sensing fundamentals and radiometry. United Kingdom: Artech House, 1981, pp.1282-1450.

[2] F. Grum, R. J. Becherer, Optical radiation measurements. Volume 1 radiometry. New York: Academic Press, 1979, pp. 40-100.

[3] S. Diridollou, V. Vabre, M. Berson, L. Vaillant, D. Black, J. M. Lagarde , J. M. Grégoire, Y. Gall, F. Patat, "Skin ageing: changes of physical properties of human skin in vivo," International Journal of Cosmetics Science., vol. 23, pp. 353-362, 2001.

[4] A. Firooz, B. Sadr, S. Babakoohi, M. S. Yazdy, F. Fanian, A. K. Timsar, M. N. Kashani, M. M. Naghizadeh, Y. Dowlati, "Variation of biophysical parameters of the skin with age, gender, and body region," The Scientific World Journal., vol. 2012, pp. 1-5, 2012.

[5] R. O. Potts, E. M. Buras, D. A. Chrisman, "Changes with age in the moisture content of human skin," J Invest Dermatol., vol. 82, pp. 97-100, 1984.

[6] S. W. Harmer, S. Shylo, M. Shah, N. J. Bowring, A.Y.Owda, "On the feasibility of assessing burn wound healing without removal of dressings using radiometric millimetre-wave sensing," Progress In Electromagnetics Research., vol. 45, pp. 173-183, 2016.

[7] H . Gray, Anatomy of the human body. Philadelphia: Lea \& Febiger, 1981, pp. 100-450.

[8] P. F. Millington, R. Wilkinson. Skin (biological structure and function). United Kingdom: Cambridge University Press, 2009, pp. 48-80. 